\title{
DOCENCIA E INTERDISCIPLINARIEDAD: DICCIONARIO, BASE DE DATOS Y RECURSO INTERACTIVO SOBRE CONCEPTOS DE LINGÜÍSTICA ORIENTADOS AL GRADO DE TRADUCCIÓN E INTERPRETACIÓN
}

\section{TEACHING AND INTERDISCIPLINARITY: DICTIONARY, DATABASE AND INTERACTIVE RESOURCE ON CONCEPTS OF LINGUISTICS AIMED AT TO THE DEGREE OF TRANSLATION AND INTERPRETING}

\author{
Prof. Dr. Alfonso Zamorano Aguilar, Prof. Dr. Ezequiel Herruzo Gómez \\ D. Sergio Rodríguez Tapia, D. Pablo Castro Lora, Dña. María Aragón Segura, \\ Dña. Sara González de la Orden, Dña. Pasión Guijarro Herrador, Dña. Marina Molinos \\ Dña. Laura Palma Arroyo, D. Gonzalo Uceda Azañón \\ azamorano@uco.es/el1hegoe@uco.es \\ Universidad de Córdoba
}

Received: 01/07/2017

Accepted: 04/12/2017

\begin{abstract}
The incorporation of ICT in all environments and everyday aspects in personal and professional development suggest the pertinence of having a tool that allows on-line consultation of information on the part of students, the registration of the consulted information and the generation of reports on system activity. The present work shows a teaching tool in web system format with certain security characteristics, information integrity and accredited access to different types of users. It is a web environment that develops and includes the basic technical vocabulary (terms and concepts) that a student of the Degree of Translation and Interpretation needs for the study and learning of the subject "Linguistics" of the first course of this degree. It includes terms, definitions, exercises and basic bibliography.
\end{abstract}

Keywords: Linguistics, ICT, teaching, linguistic terminology, on-line dictionary.

\section{Resumen}

La incorporación de las TIC en todos los entornos y aspectos cotidianos tanto en el desarrollo personal como profesional, plantean la pertinencia de disponer de una herramienta que permita la consulta on-line de información por parte del alumnado, el registro de la información consultada y la generación de informes sobre la actividad del sistema. En el presente trabajo se muestra una herramienta docente en formato sistema web con determinadas características de seguridad, integridad de la información y acceso acreditado a distintos tipos de usuarios. Se trata de un entorno web que desarrolla e incluye el vocabulario técnico (términos y conceptos) básicos que un estudiante del Grado de Traducción e Interpretación necesita para el estudio y aprendizaje de la asignatura "Lingüística" del primer curso de dicho Grado. Incluye términos, definiciones, ejercicios y bibliografía básica.

Palabras clave: Lingüística, TIC, docencia, terminología lingüística, diccionario on line

\section{INTRODUCCIÓN}

En el presente artículo presentamos los resultados de un proyecto de innovación educativa incardinado en el marco del Plan de Innovación y Buenas Prácticas Docentes de la Universidad de Córdoba (curso 2016-2017), ya que pretende promover la innovación en la metodología docente en Lingüística, tanto en clases teóricas como prácticas, mediante la incorporación de las tecnologías de la información y la comunicación al proceso de enseñanzaaprendizaje de la materia indicada.

Asimismo, dentro de una docencia en los Grados basada en el desarrollo de las competencias estudiantiles, este proyecto se focalizó en dos de las líneas prioritarias de la modalidad 1 del citado Plan: el trabajo por competencias y la transferencia del conocimiento teórico a la práctica. No obstante, la prioritaria es el trabajo por competencias.

El objetivo primordial del proyecto consistió en el diseño de un diccionario que aglutine todos los conceptos básicos tratados en la asignatura de Lingüística, materia de carácter básico impartida en el primer curso del Grado de Traducción e Interpretación, si bien el resto de alumnado de los grados en los que se imparten contenidos lingüísticos pueden beneficiarse del recurso, así como los doctorandos que trabajen en las líneas de investigación de lingüística y materias afines.

La principal ventaja que presenta el recurso que aquí proponemos radica en que permite la actualización constante de la información que contiene la base de datos (de forma que se evita la concepción estática de un diccionario en papel) y constituye un entorno apto para el correcto seguimiento del alumnado a través de una plataforma personalizada, en la que el alumnado puede aportar información sobre la dificultad que encuentra en el estudio de los diferentes conceptos, seguir su evolución o realizar comentarios y sugerencias. 
Entre los principales diccionarios sobre lingüística que existen, pueden enumerarse algunos publicados en los últimos quince años, los más actualizados internacionalmente en el ámbito de la lingüística. Estos volúmenes, en papel, tienen una clara orientación terminológica, ya que pretenden ser compendios dirigidos a especialistas o semiespecialistas, pero no especificamente alumnado novel. Se trata, por tanto, de textos que el alumnado debe consultar pero que serán más comprensibles si, previamente, han resuelto dudas básicas a través del diccionario que nosotros planteamos. Así, el manejo de fuentes bibliográficas como las que siguen se realizará de forma más eficiente y significativamente comprensiva:

- Enrique Alcaraz VARÓ y María Antonia MARTíneZ LinARES (eds.) (2004): Diccionario de lingüistica moderna. Barcelona: Ariel.

- R.L. TRASK; Peter STOCKWELL (ed.) (2007): Language and linguistics: the key concepts. Abingdon, UK [etc.]: Routledge.

— Elizabeth Luna Traill, Alejandra Vigueras Ávila, Gloria Estela BAEZ PINAL (eds.) (2007): Diccionario básico de lingüistica. México: Universidad Nacional Autónoma de México.

— David CRYstal (2010): The Cambridge encyclopedia of language. Cambridge; New York: Cambridge University Press.

- Franck Neveu (2011): Dictionnaire des sciences du langage. Paris: Colin.

- Hartmut HeuERMAnN (2014) Sprachwissenschaft für den Alltag: ein Kompendium. Frankfurt: Peter Lang.

Otros recursos, siguiendo en el ámbito de la Lingüística, tienen un carácter didáctico, tanto en soporte papel como digital:

— Ángel Alonso-CORTÉs (2002): Ejercicios de lingüística [Recurso electrónico]. Public. Madrid: Visor Libros.

— Frank PARKER, Kathryn RILEY (eds.) (2005): Linguistics for non-linguists: a primer with exercises. Boston: Pearson.

- William O'Grady, John ArChibAld, Mark ARONOFF, Janie REES-Miller (eds.) (2010): Contemporary linguistics: an introduction. Boston, Mass.: Bedford-St. Martins ${ }^{1}$.

— Lidia TAillefer DE HAYA \& Rosa MuÑoz LunA (Eds.) (2012): Glosarios lingüísticos digitales [Recurso electrónico]. Málaga: VG Ediciones, D.L.

Más cercanos al producto que presentamos, se encuentran algunas bases de datos sobre bibliografía lingüística (como la Linguistic Bibliography Online) o glosarios y diccionarios especializados en un ámbito (como el Diccionario de términos clave de ELE del Centro Virtual Cervantes, editado por Ernesto Martín Perís). No obstante, escasean los recursos didácticos e interactivos sobre lingüística y no existe ninguno, que conozcamos, que permita crear un sistema en el que el alumnado pueda acceder a registros sobre su profesorado y participar en la propia base de datos a través de sus comentarios.

Así pues, el diccionario que aquí se presenta tiene la forma de base de datos, alojada en un sitio web específico para el recurso, y agutina las actividades interactivas y los recursos documentales necesarios para que el alumnado pueda desarrollar su proceso de aprendizaje de forma óptima y, tras el análisis y explicaciones de clase, pueda desarrollar también dicho aprendizaje de manera autónoma.

Como resulta conocido, la Lingüística es una materia esencial en los grados que abordan lenguas en las universidades en general y en la de Córdoba en particular. Concretamente, en el Grado de Traducción e Interpretación, casi la todas las asignaturas hunden sus raíces en los fundamentos teóricos y metodológicos de la Lingüística, lo que la convierte en una asignatura descriptiva y explicativa de los fenómenos y problemas a los que el alumnado tendrá que poner solución a lo largo de su formación universitaria. La densidad de contenidos junto con la abstracción de ciertos conceptos, así como la interrelación que existe entre los diferentes temas y sistemas conceptuales del temario, exigen que el alumnado realice un esfuerzo adicional por ordenar, relacionar y organizar dichos conceptos, de forma que al finalizar la asignatura sea capaz de a) transmitir con fluidez y naturalidad los contenidos especializados a cualquier tipo de público, y b) aplicar los conceptos a la resolución de problemas comunicativos.

Si bien a lo largo de la materia cada tema circunscribe su propio sistema conceptual y lo relaciona con lo analizado anteriormente, de forma que se sigue un orden riguroso que garantice el correcto seguimiento de la materia, mediante la graduación de la dificultad de los conceptos, se considera que la creación de un recurso interactivo permita organizar estos conceptos de forma versátil, intuitiva y dinámica facilitaría la labor de aprendizaje del alumnado, ya que podría evaluar su desarrollo formativo a través de las actividades que se asocian a cada tema.

\footnotetext{
${ }^{1}$ En concreto, el manual de O’Grady et al. (2010) es uno de los más empleados en el contexto internacional por la sección de actividades y de recomendaciones que existe al final de cada capítulo.
} 
Este recurso no acaba con el proceso tradicional seguido hasta ahora de exposición temática en seminarios magistrales, sino que se trata de un complemento a las exposiciones de clase, que podrá ser empleado en las horas no presenciales de la asignatura.

Además, por el propio diseño de las actividades de evaluación de la asignatura en el Grado de Traducción e Interpretación de la Universidad de Córdoba, el alumnado tiene que someterse a la exposición oral de conceptos seleccionados por el profesor en el aula. Este hecho obliga al alumnado a mantener una atención específica a la claridad conceptual de la materia y a su correcta formulación y relación. La herramienta que presentamos permitirá asentar de forma dinámica e interrelacionada los conceptos en la docencia tanto presencial como no presencial, ya que de manera natural el alumnado emplea el ordenador, tanto en clase como en sus horas de estudio individualizado fuera del aula. Además, este instrumento lexicográfico innovador permite focalizar y reforzar los contenidos teóricos explicados en clase a través de ejercicios de aplicación de diversa naturaleza: preguntas de opción múltiple, verdadero y falso, relación de conceptos, relleno de huecos, cuestiones de respuesta breve, etc. que incluyen la autoevaluación y repetición como funcionalidades específicas del diccionario.

Además, está prevista, en una fase posterior, la transferencia a una app, lo que permitiría un acceso a la teoría y a la práctica a través de otros dispositivos electrónicos, como el móvil.

Como puede observarse, la herramienta que presentamos en este artículo, por tanto, incide en varias de las competencias fundamentales para los estudios de Grado según establece el Real Decreto 861/2010, de 2 de julio, por el que se modifica el Real Decreto 1393/2007, de 29 de octubre, por el que se establece la ordenación de las enseñanzas universitarias oficiales. Disponible en: https://www.boe.es/buscar/doc.php?id=BOE-A-2010-10542. Como se ha indicado, una de las competencias esenciales que se pretende desarrollar es el acceso y manejo de información a través de TIC. De hecho, la incorporación de las TIC en todos los entornos y aspectos cotidianos tanto en el desarrollo personal como profesional, plantean la pertinencia de disponer de herramientas que permitan la consulta on line de información por parte del alumnado, el registro de la información consultada y la generación de informes sobre la actividad del sistema.

Desde el punto de vista técnico, este diccionario se compone, básicamente, de un sistema web con determinadas características de seguridad, integridad de la información y acceso acreditado a distintos tipos de usuarios. Además, en principio, se plantea que dicho sistema sea realizado con herramientas de libre disposición y sea un sistema "responsivo" (adapta las dimensiones del contenido y muestra los elementos de una forma ordenada y optimizada) que permita acceder al mismo de forma cómoda no solamente desde un ordenador conectado a internet, sino también desde cualquier dispositivo móvil con conexión de datos. Asimismo, se pretende que el sistema cumpla con los requisitos y normativa de accesibilidad y diseño.

\section{OBJETIVOS}

Los objetivos se plantean con la finalidad de propiciar el desarrollo de las competencias indicadas y el aprendizaje comprensivo y dinámico de la Lingüística (en el caso del alumnado al que se aplica) y, además, estos objetivos también pretenden fomentar (en el caso del alumnado que ha participado en el grupo de trabajo del proyecto) el trabajo interdisciplinar y de iniciación a la investigación en equipo.

Los objetivos, pues, se concretan en los siguientes:

\section{Objetivos relacionados con la materia Lingüística y con las competencias disciplinares/docentes:}

- Ofrecer una herramienta web interactiva que sintetice y ordene el sistema conceptual del temario de Lingüística del Grado de Traducción e Interpretación de la Universidad de Córdoba, en particular.

- Trabajar competencias fundamentales en los estudios de Grado: capacidad de síntesis, transferencia de la teoría a la práctica/aplicación, uso de TIC, autoaprendizaje y autoevaluación.

— Incidir en una docencia que fomente la virtualización y el acceso al conocimiento de forma dinámica, interactiva y aplicada.

- Reunir de forma rigurosa y ordenada la bibliografía relacionada con cada concepto, de forma que pueda servir para su formación en los primeros años y para futuras consultas especializadas en su etapa profesional o investigadora (por ejemplo, durante la elaboración del TFG o TFM).

- Presentar un entorno en el que el alumnado pueda poner a prueba sus conocimientos con ejercicios interactivos, tanto de carácter teórico como de carácter aplicado.

- Ofrecer un sistema en el que el alumnado pueda seguir su evolución durante la asignatura a través de un registro atomatizado, individual y personalizado.

- Permitir que el alumnado pueda participar en su proceso de aprendizaje, determinando la dificultad de los conceptos con los que trate e iniciando un proceso de retroalimentación con la plataforma y el profesorado. 
- Brindar al alumnado y al profesorado de otros grados en los que se imparte Lingüística la posibilidad de fomentar competencias tecnológicas, de autoaprendizaje y autoevaluación en el estudiantado a través de la teoría y la práctica de la materia Lingüística.

\section{Objetivos relacionados con la herramienta:}

Desde el punto de vista de la herramienta, nos planteamos una serie de objetivos como solución a requisitos funcionales, no funcionales y de interfaz:

- Ofrecer al alumnado y al profesorado de otros grados en los que se imparte Lingǘstica la posibilidad de fomentar competencias tecnológicas, de autoaprendizaje y autoevaluación en el estudiantado a través de la teoría y la práctica de la materia Lingüística.

- Poder acceder al sistema informático desde cualquier ordenador conectado a internet de forma segura.

- Permitir a los/as usuarios/as conectados/as al sistema interactuar con la base de datos alojada en un servidor dedicado. De esta forma se garantiza la seguridad e integridad de los datos recogidos en el sistema.

- Almacenar la información por medio de cuestionarios dinámicos en una base de datos MySQL, consiguiendo robustez, rapidez y estabilidad a la hora de la recogida y estudio posterior de los datos introducidos.

- Conseguir seguridad de acceso: el sistema debe proteger los datos contra accesos no autorizados.

— Obtener integridad de los datos: el sistema debe verificar toda la información almacenada.

- Lograr la fiabilidad del sistema: debe responder contra posibles errores por parte del usuario o por el propio sistema.

- Diseñar un sistema capaz de manejar aquellos errores que puedan producirse durante su funcionamiento de manera correcta, corrigiendo en la medida de lo posible sus causas, minimizando sus consecuencias e informando a los usuarios de manera exacta y clara sobre el error producido y sus características.

Además de los anteriores, en cuanto a los requisitos de interfaz, el sistema se ha desarrollado de forma que sea sencillo de utilizar, incluyendo todos los datos e interpretando las salidas, y manteniendo su consistencia en el diseño (mismas reglas de funcionamiento desde el punto de vista del usuario).

\section{MATERIAL Y MÉTODOS}

Esta herramienta ha requerido trabajar en dos fases bien diferenciadas:

\section{Fase de preparación terminográfica:}

- Se ha partido de la selección de conceptos más relevantes dentro del temario de la asignatura. Se han incluido un total de 390 conceptos.

- Se ha procedido a la identificaicón de estos conceptos dentro de la bibliografía especializada y han esbozado breves ensayos o definiciones sobre cada concepto, al que acompañan las referencias bibliográficas y las recomendaciones bibliográficas complementarias.

- Se han revisado y verificado los conceptos por especialistas en la materia y por el profesorado que imparte la asignatura.

- La última tarea ha consistido en la asociación de ejercicios conceptuales y aplicados (de tipología variada según se ha expuesto) a cada concepto, así como la asociación de ejercicios de reflexión.

- Cada entrada de la base de datos contiene diversos campos relacionados, como el tema al que pertenece el texto, el ámbito científico en el que se inscribe, los conceptos relacionados, el autor o corriente científica de la que surge, etc. De esta forma, el alumnado podrá navegar usando diferentes filtros a través del recurso.

- Se ha creado un sitio web específico que aloja los contenidos y ejercicios interactivos (vid. apartado de resultados de este artículo).

- Se ha creado un sistema de registro de actividad de los usuarios, que incluye los temas más consultados, las actividades realizadas con el porcentaje de aciertos y errores y las recomendaciones del sistema.

\section{Fase de intervención informática:}

1. Preparación: Esta fase ha comprendido las tareas y acciones para adquirir los conocimientos y experiencia necesaria para abordar e iniciar la ejecución del proyecto de forma correcta. Entre las tareas que se han realizado figuran, en principio: establecer un punto de partida para iniciar el proyecto, decidir lenguajes y entornos de 
programación, el estudio de las herramientas para la creación del sistema, estudio de metodologías y procesos, etc. La finalidad fue la adquisición de los conocimientos suficientes para la toma de decisiones que se deben llevar a cabo, tanto en el análisis, diseño e implementación.

2. Análisis: en esta fase se ha realizado la recogida de la información necesaria para la implementación del sistema y el servicio web que se ha construido, así como de la información sobre el problema al que se ha dado solución.

3. Diseño: tras realizar el análisis, se ha llevado a cabo el diseño del sistema, distribuyendo las funcionalidades previstas de la forma más óptima posible, proporcionando un entendimiento fácil e intuitivo, así como su mantenimiento. En esta fase el trabajo interdisciplinar de lingüistas (o estudiantes de lingüística) e informáticos (o estudiantes de informática) ha resultado esencial, pues ha permitido poner en común sugerencias, ideas o conceptos en beneficio de la herramienta final.

4. Implementación: desarrollo e implementación del sistema sobre la base del análisis y diseño del mismo definidos en las etapas anteriores: 1) la implementación de la estructura del sistema planteada en la descripción del dominio de la información; 2) a continuación, desarrollo de la lógica del programa de forma que se alcance toda la funcionalidad requerida; 3) la implementación del sistema se ha llevado a cabo de manera iterativa, realizando tanto partes de interfaz como de lógica simultáneamente; 4) el sistema se ha desarrollado haciendo uso del framework Joomla y acceso a base de datos MySQL.

5. Prueba: en esta fase hemos sometido al sistema a pruebas exhaustivas para comprobar que la aplicación funcionaba de manera correcta y producía los resultados deseados. Debido a la naturaleza de un desarrollo iterativo también se han hecho pruebas durante el proceso de desarrollo para evitar errores e intentar simplificar las labores de validación y verificación. Estas pruebas han sido tanto estructurales como funcionales.

\section{RESULTADOS OBTENIDOS Y DISCUSIÓN}

Como resultado final de desarrollo del proyecto, se ha implementado un sistema web con la funcionalidad prevista totalmente desarrollada. El sistema se ubicará en la dirección URL: www.uco.es/sialin (Sistema Interactivo Aplicado de Lingüística).

El sistema cuenta con cuatro tipos de perfil, divididos en dos grupos. El primer grupo se corresponde con los usuarios visitantes. Estos usuarios no se registrarán en el sistema, teniendo por tanto un acceso limitado al mismo. El segundo grupo lo componen tres tipos de usuarios: usuarios alumnos, usuarios docentes y usuarios administradores. Los usuarios alumnos podrán realizar pruebas de evaluación. Los docentes podrán gestionar dichas pruebas. Por último, los administradores podrán organizar y manipular la información contenida en el sistema.

Todos los usuarios gozan de diversos niveles de acceso y tipos de casos de uso asociados. Los usuarios visitantes solo podrán consultar el glosario de términos. Los usuarios registrados podrán, además, autoevaluarse mediante pruebas tipo test y de respuesta corta. Estas pruebas serán evaluadas por los docentes. También podrán introducir valoraciones sobre la información almacenada en el glosario.

Los usuarios administradores tendrán acceso a los casos de uso de gestión de la información. Podrán editar la información asociada a los usuarios registrados, gestionar las entradas del glosario y administrar las pruebas disponibles. Este modelo de casos de uso se corresponde con la Figura 1.

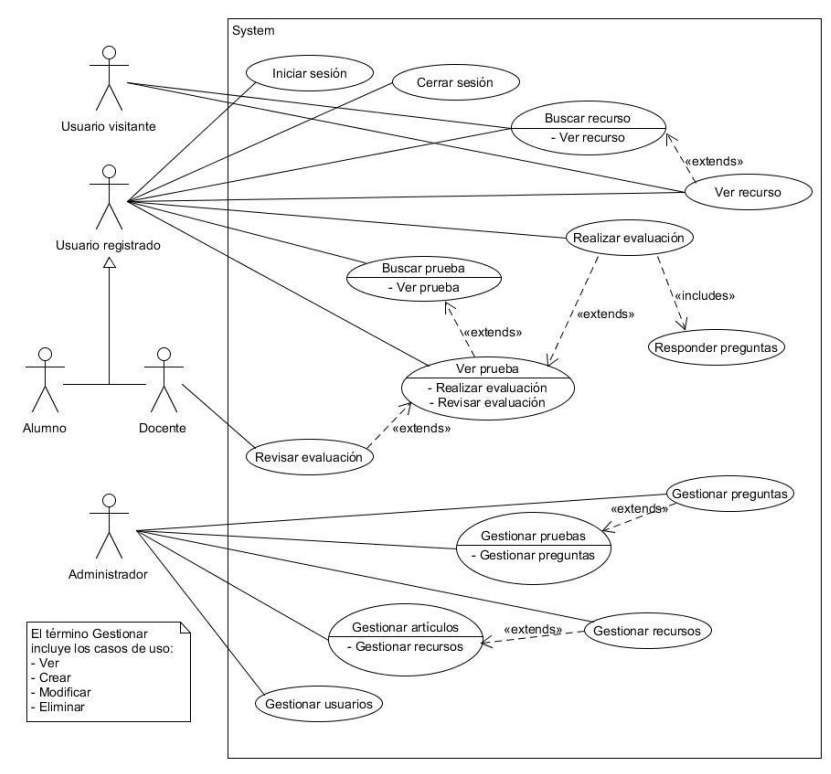


El modelo de datos utilizado para responder a las necesidades de información del proyecto se muestra en la Figura 2. El sistema empleará un registro de usuarios, para el cual almacena información adicional que permite su catalogación.

información sobre primera de ellas es la evaluación de las someterán los relacionan con una usuario, al responder autoevaluación, que información más será la relacionada En el modelo se términos, con un relaciones entre sí y atributos. De esta podrá mostrarse una información.

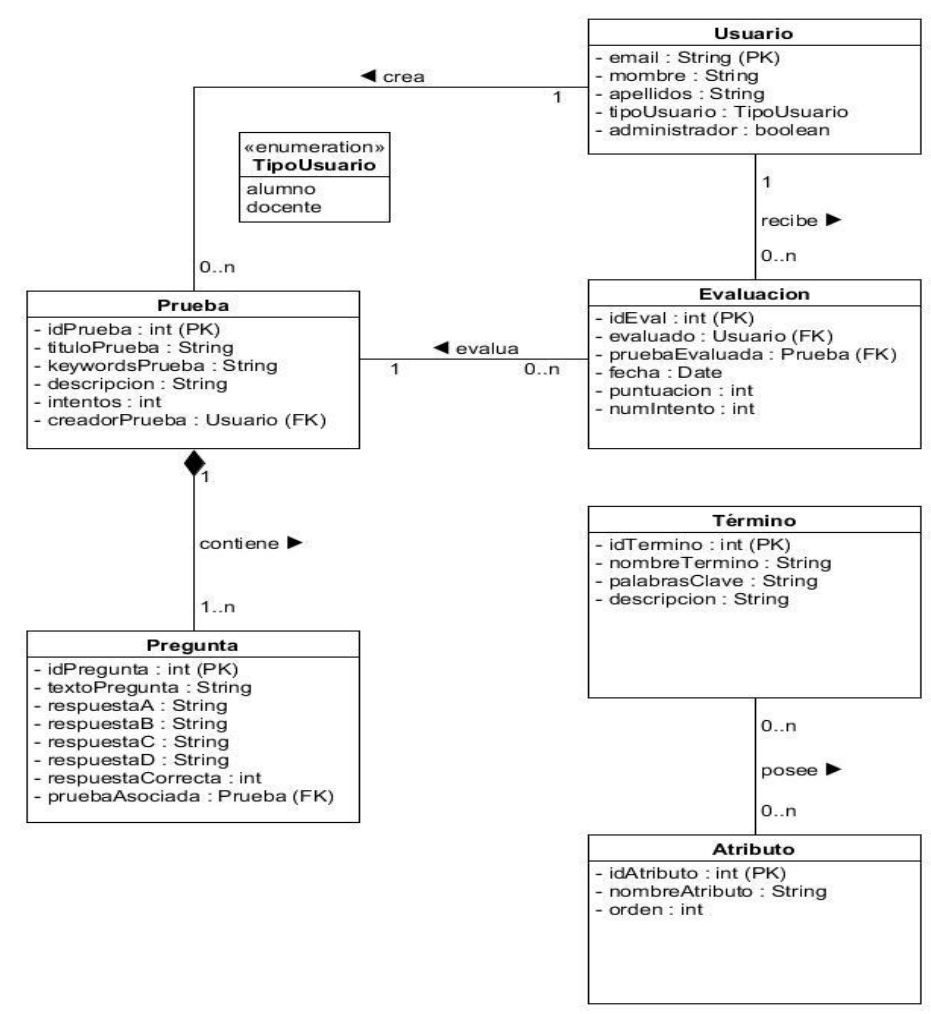

También acumula dos áreas fundamentales. La correspondiente a la pruebas a las que se usuarios. Las pruebas se serie de preguntas. Cada a la prueba, generará una será también almacenada. La básica que se almacenará con el glosario de términos. guardará cada uno de los número variable de un listado flexible de forma, para cada término, cantidad personalizable de 
Respecto a la interfaz del sistema, A continuación, se muestran varias imágenes correspondiendo a pantallas de usuario que permiten la navegación entre sus diversas funcionalidades de forma clara e intuitiva. Se mostrarán de forma resumida indicando en el título la funcionalidad correspondiente.

1) PANTALLA PRINCIPAL. Apariencia del sistema antes de iniciar sesión, visible para cualquier usuario y acceso al sistema. Interfaz que permite introducir usuario y contraseña para entrar en el sistema (opción "Iniciar sesión”):

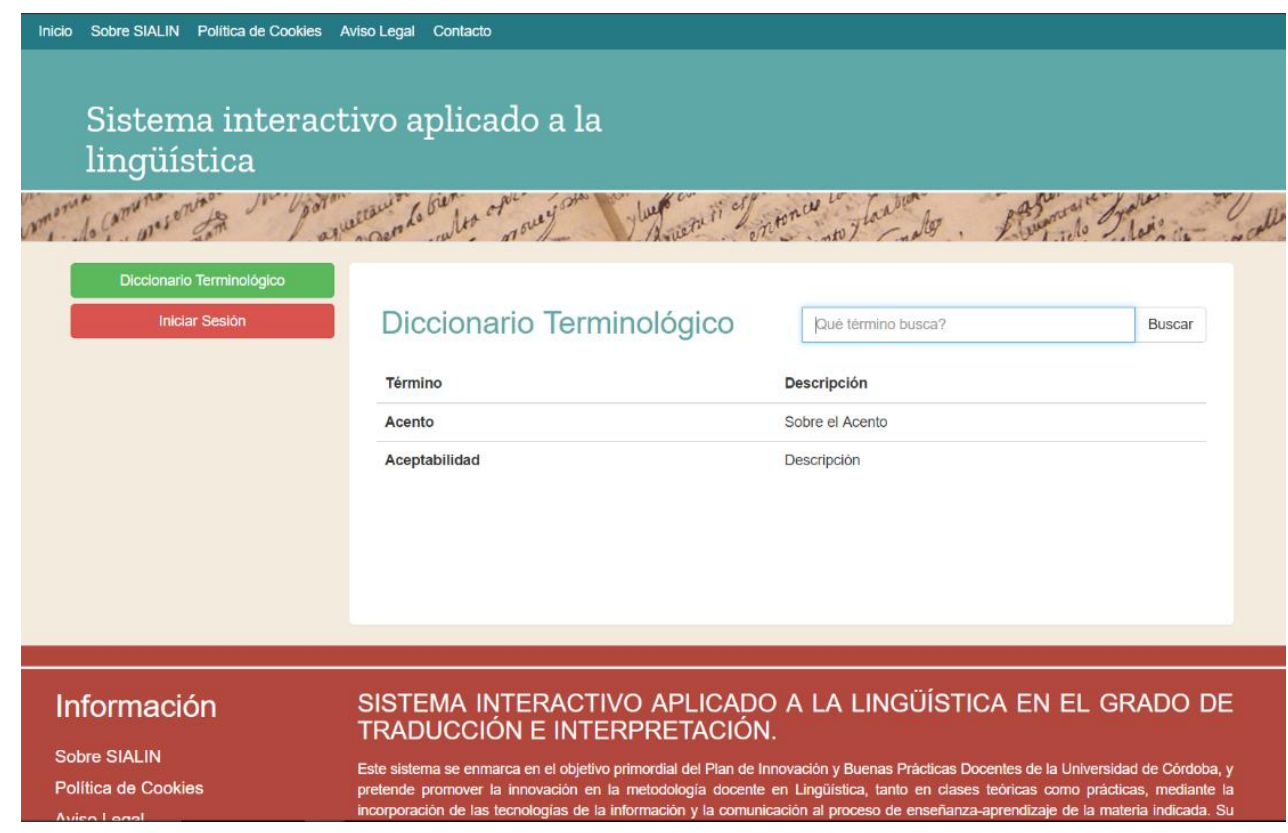

2) PANTALLA PRINCIPAL CON SESIÓN INCIADA COMO ALUMNO

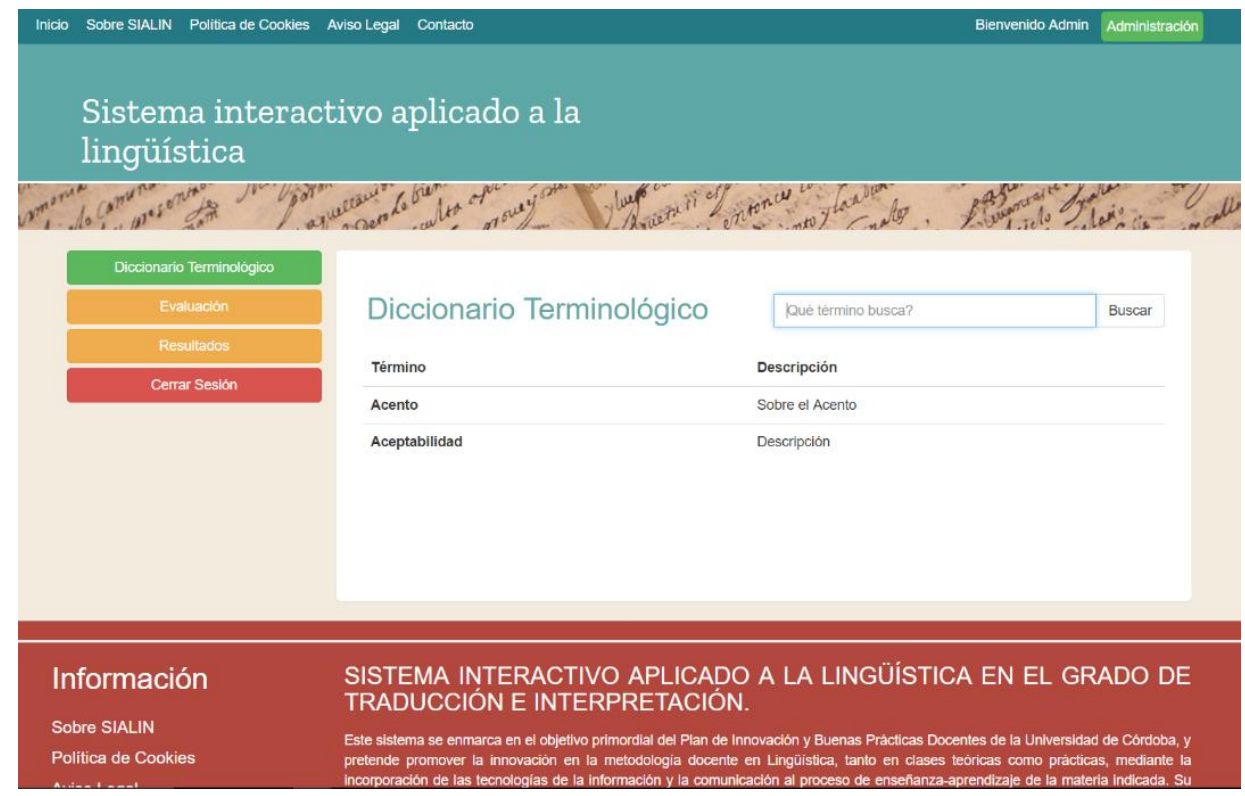

3) PANTALLA PRINCIPAL CON SESIÓN INCIADA como administrador 


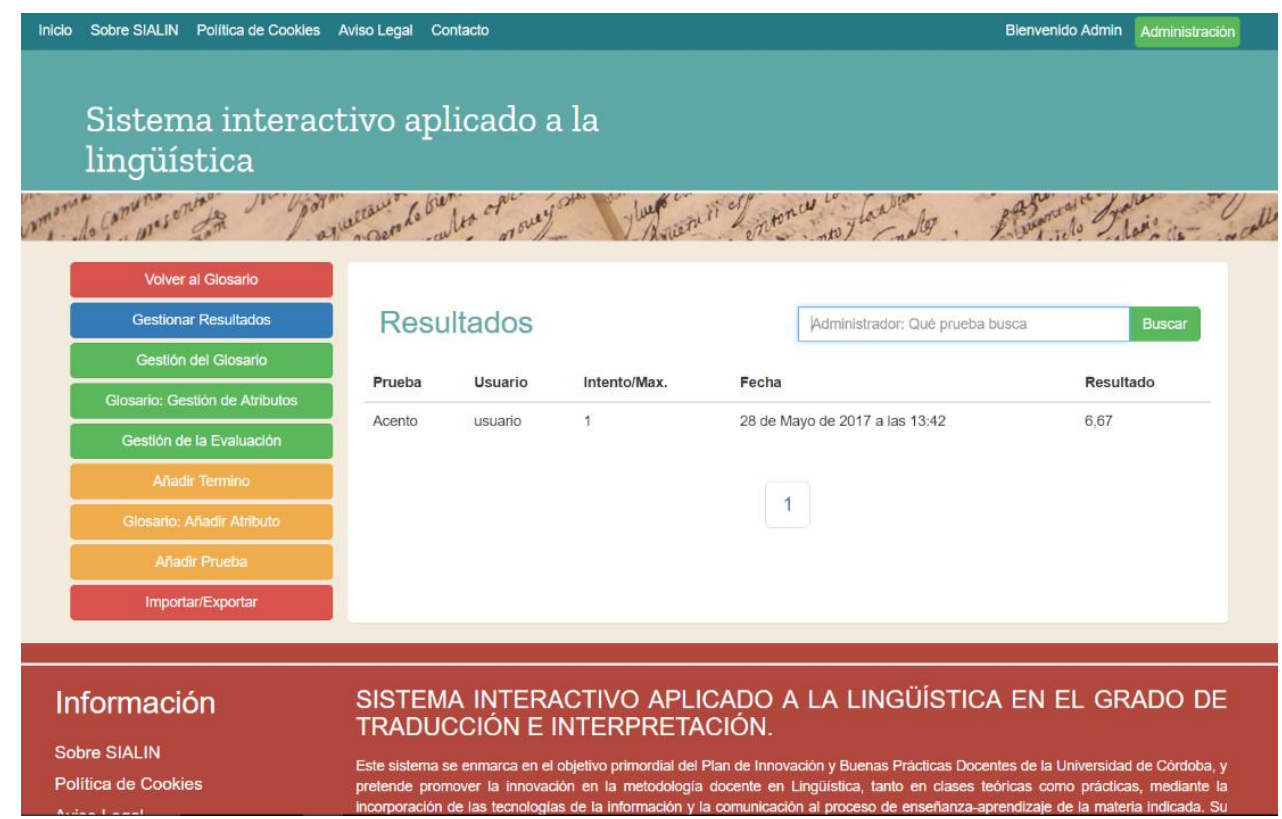

4) INSERCIÓN, MODIFICACIÓN Y/O EDICIÓN DE UN TÉRMINO

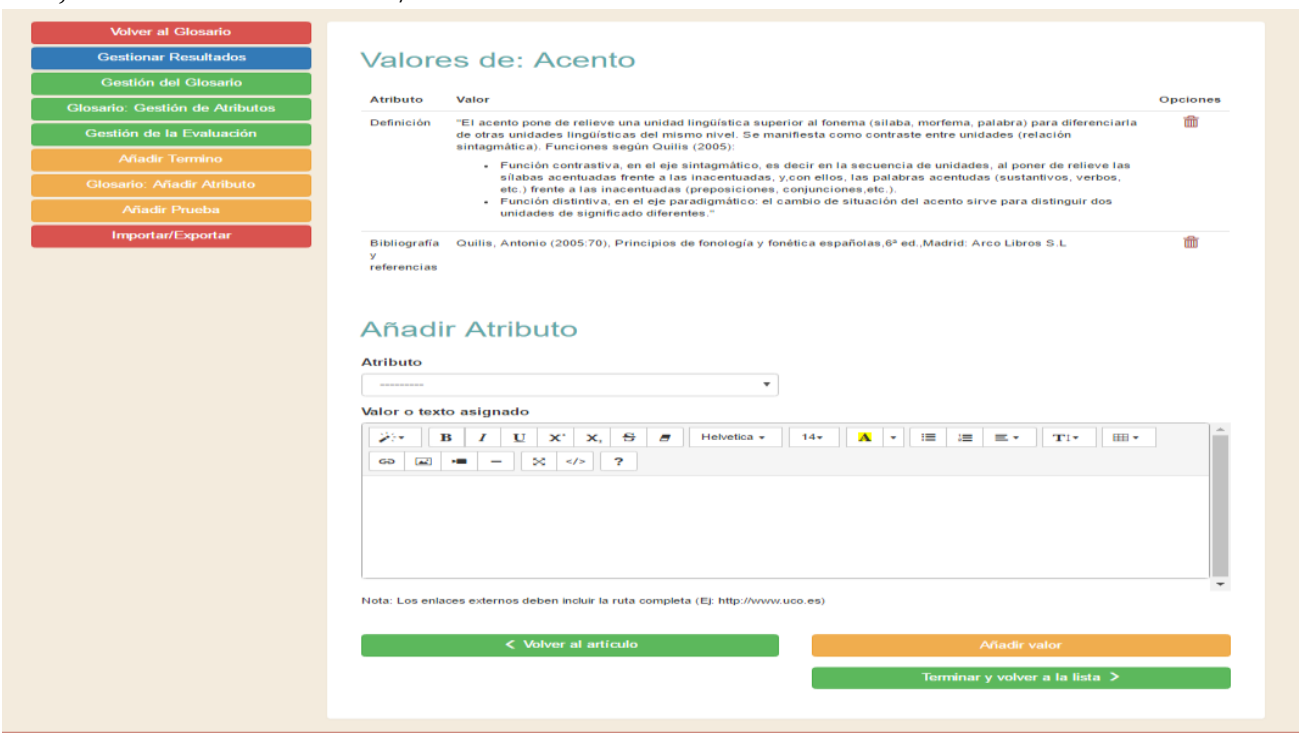

5) RESULTADO BUSQUEDA DE UN TERMINO y valoración del mismo

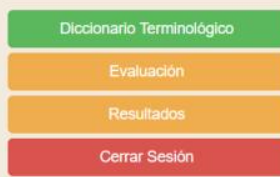

Diccionario Terminológico

\begin{tabular}{|l|l|}
\hline Què tèrmino busca? & Buscar \\
\hline
\end{tabular}

Acento

Definición

"El acento pone de relieve una unidad linguiistica superior al fonema (silaba, morfema, palabra) para diferenciarla de otras unidades lingüisticas del mismo nivel. Se manifiesta como contraste entre unidades (relación sintagmática). Funciones segùn Quilis (2005):

- Función contrastiva, en el eje sintagmático, es decir en la secuencia de unidades, al poner de relieve las silabas acentuadas frente a las inacentuadas, y,con ellos, las palabras acentudas (sustantivos, verbos, etc.) frente a las inacentuadas (preposiciones, conjunciones, etc.).

bio de situación del acento sirve para distinguir dos unidades de

Bibliografía y referencias

Quilis, Antonio (2005:70), Principios de fonologia y fonética españolas, $6^{a}$ ed,,Madrid: Arco Libros S.L

Pruebas:

- Acento

Utilidad

Complejidad

Nivel de desarrollo

3

3

3

Enviar valoración 


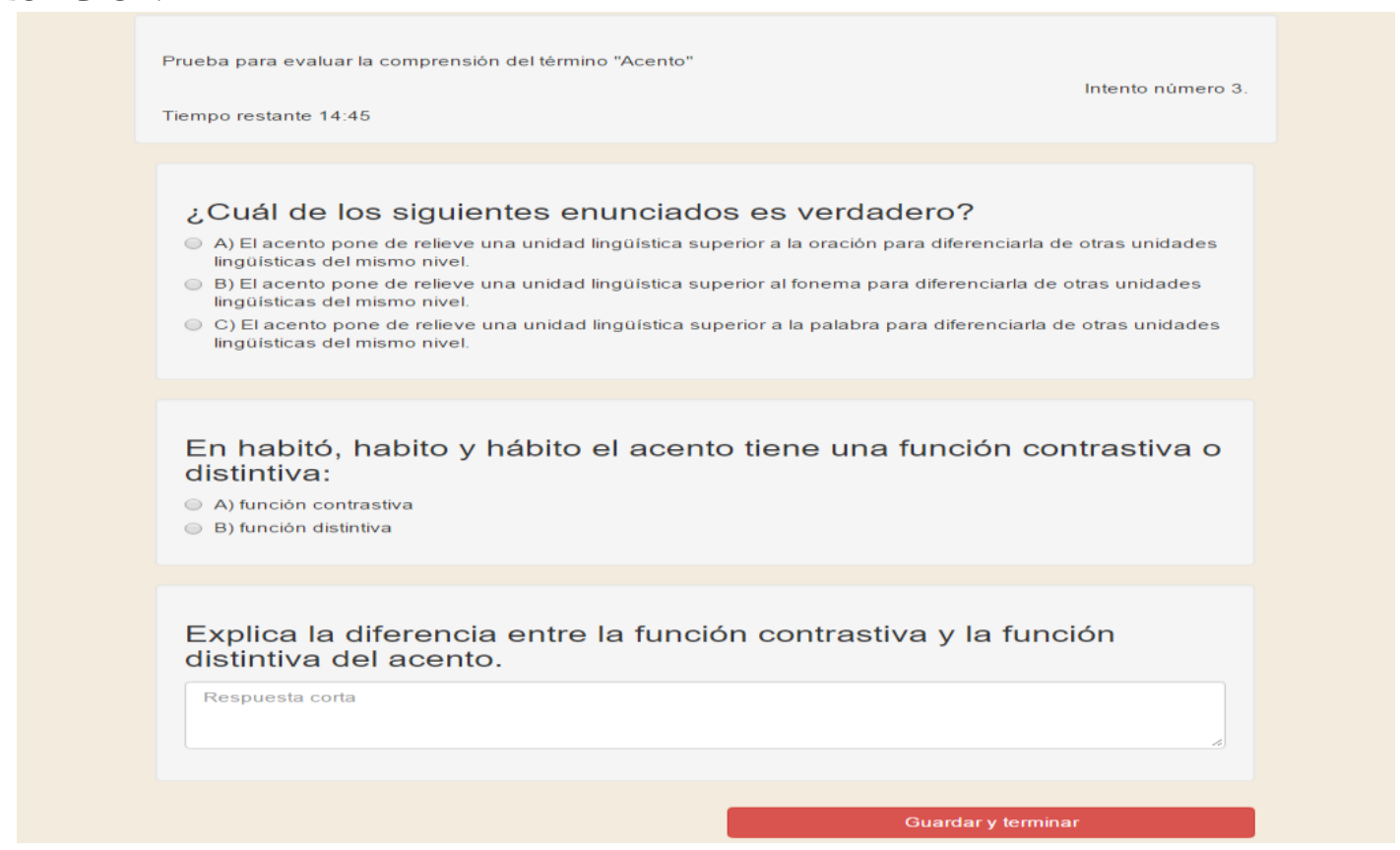

8) RESULTADOS

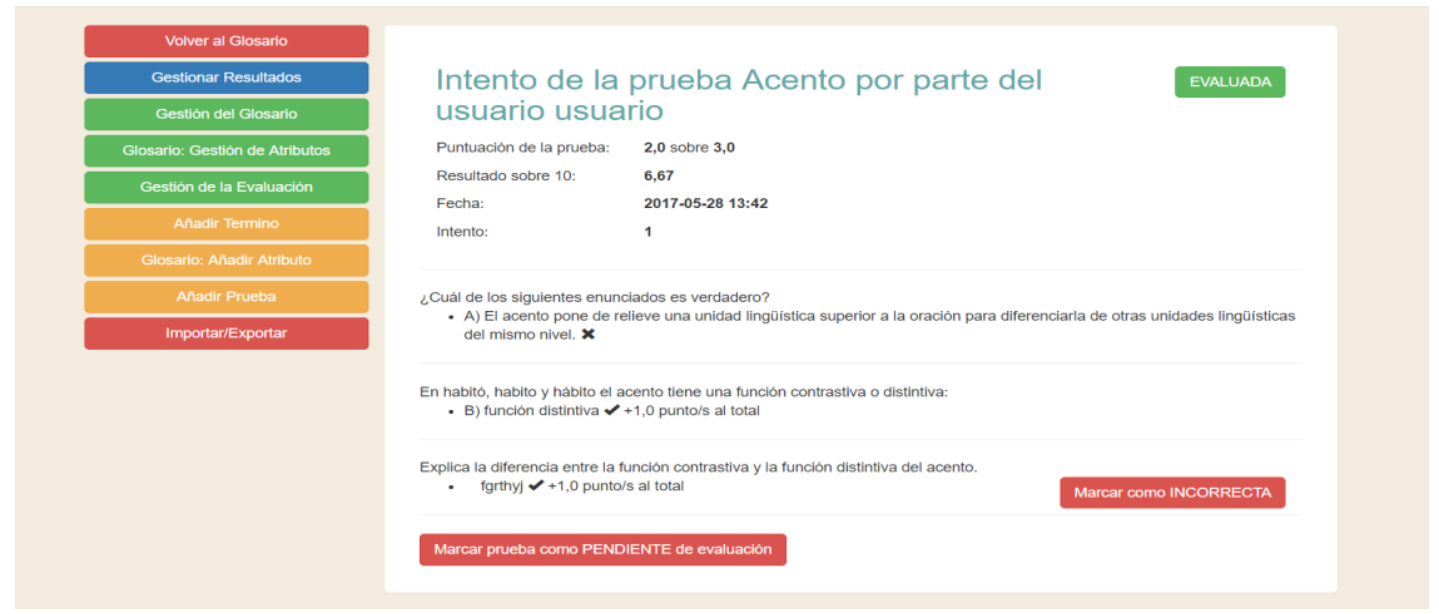

9) Importar/exportar. Permite actualizar la base de datos con nuevos términos de forma automática desde un fichero en formato excel (que fácilmente puede ser creado desde cualquier otra base de datos) o exportar la informacón contenida en la base de datos. 


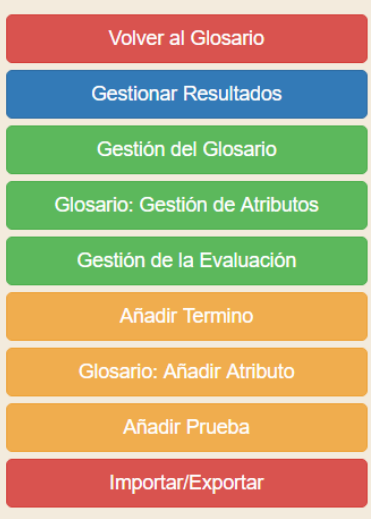

\section{Importar/Exportar}

\section{Importar BBDD desde fichero .xlsx}

ATENCIÓN: si continúa con esta operación reemplazará todos los datos almacenados por los del nuevo libro excel. Asegurese de realizar una copia de seguridad con la opción Descarga fichero Excel antes de continuar.

File:

Seleccionar archivo Ningún archivo seleccionado

Importar fichero Exce

Exportar BBDD a fichero .xIsx

Otros resultados obtenidos con el proyecto desarrollado:

-En el personal de proyecto. Dado que una parte del personal del proyecto es personal en formación y alumnado colaborador del área de Lingüística General de la Universidad de Córdoba y del Grado de Ingeniería Informática, se ha prentenddio que este personal trabaje en equipo y de forma supervisada y coordinada en el marco de un proyecto interdisciplinar lingüística-informática. Esta formación adicional a la que reciben en su grado les ha permitido ir adquiriendo valores positivos hacia la investigación y, de forma particular, hacia su inminente TFG o Tesis Doctoral.

-En el alumnado de la(s) asignatura(s) implicada(s). En el alumnado de Traducción e Interpretación (170 cada curso) y del resto de grados lingüístico-literarios (unos 1000 estudiantes más) se ha pretenddio mejorar la asimilación y estudio de la asignatura y el óptimo desarrollo de competencias tecnológicas y de gestión de la información, así como aquellas que tienen que ver con la aplicación y transferencia del conocimiento.

\section{CONCLUSIONES}

Este sistema, desarrollado al amparo del Plan de Innovación y Buenas Prácticas Docentes de la Universidad de Córdoba, es una herramienta sencilla de utilizar tanto por el alumnado como por el profesorado/administrador del sistema. Aunque no se han hecho pruebas de funcionamiento real con el alumnado que asiste y participa en clase, las pruebas tanto de funcionamiento interno como de uso por parte de alumnos de prueba con características similares a las que lo utilizarán han sido satisfactorias. Dispone de una interfaz sencilla de utilizar por cualquier tipo de usuario y una funcionalidad completa para satisfacer los objetivos propuestos desde el inicio del proyecto. Las pruebas reales con alumnado cursando la asignatura de Lingüística se realizarán el próximo curso académico (curso 2017-2018), donde se podrán concluir la efectividad del sistema y determinar si cumple con sus objetivos formativos.

\section{BIBLIOGRAFÍA}

Wiegers, K. \& Beatty, J. (2013). Software requirements (3rd ed.). Redmond: Microsoft Press.

Pressman, R. (2010). Ingeniería del software (3rd ed.). Madrid: McGraw-Hill.

Página WordReference: http://www.wordreference.com/

Página Dicciomed: Diccionario médico-biológico, histórico y etimológico: http://dicciomed.eusal.es/

https://infotra.wordpress.com/2014/11/24/dicciomed-eusal-es-diccionario-medico-biologico-historico-etimologico/

Página ILV-México: http://www.mexico.sil.org/es/publicaciones/articulacion

Página Real Academia Española: http://www.rae.es/

Página Diccionario de términos clave de ELE Centro Virtual Cervantes:

http://cvc.cervantes.es/ensenanza/biblioteca ele/diccio ele/indice.htm 